\title{
Current-Voltage Characteristic of Electrospray Processes in Microfluidics
}

\author{
V. R. Gundabala, N. Vilanova, ${ }^{*}$ and A. Fernández-Nieves \\ School of Physics, Georgia Institute of Technology, Atlanta, Georgia 30332-0430, USA \\ (Received 1 April 2010; revised manuscript received 24 June 2010; published 6 October 2010)
}

\begin{abstract}
We use a glass-based microfluidic device to study the electric current behavior of an electrospray process in the presence of a coflowing liquid. The current shows strong voltage dependence and weak flow rate dependence, in stark contrast to classical electrospray. By considering that the current is dominated by convection near the apex of the conical meniscus and driven by tangential electric stresses, we quantitatively capture the voltage and flow rate dependence of the current. Our results elucidate the influence of external field strength and open the way to achieve robust electric control of the current and of the drop size in microfluidics.
\end{abstract}

DOI: 10.1103/PhysRevLett.105.154503

PACS numbers: 47.65.-d, 47.55.db, 47.60.Kz

Since the pioneering work of Zeleny on the electrically induced deformation of a spherical liquid drop into a conical meniscus [1], the applications of electrically assisted processes have grown enormously [2] in fields like mass spectrometry [3], powder technology [4], fiber generation [5-8], and food and drug delivery $[9,10]$. This widespread use results from the evolution of the conical meniscus, which deforms into a liquid ligament or jet that either breaks into drops or stretches to form fibers. In the case of drop formation in air or vacuum, the process, which results in an aerosol or spray, is called electrospray. However, this term is also used even if the medium is a quiescent liquid bath [11] or a flowing liquid in a microfluidic device [12], where emulsions rather than aerosols are generated.

The electrospraying process results from the finite conductivity of the liquid, which promotes the migration of charges in the bulk towards the bounding interface to screen the external electric field; this is nothing but the propensity of any conductor to have a zero net field in its inside. However, since liquids are easy to deform, the normal electric field outside the liquid that results from the presence of this surface charge is able to stretch the meniscus away from its original spherical shape. This deformation is opposed by surface tension, which is responsible for exerting increasingly larger stresses as the interface progressively deviates from the favored spherical shape. The characteristic conical shape that typifies electrospray processes, often referred to as a Taylor cone, thus results from the hydrostatic equilibrium between electrical and surface tension stresses [13,14].

Despite the fact that the cone formation is driven by the applied electric field $[15,16]$, it is usually found that the scaling laws for both the electric current and the jet or drop diameter do not depend on the field strength and are solely controlled by the flow rate of the injected liquid [17-22]. Interestingly, there are situations where the opposite behavior has been observed, with the current exhibiting a marked dependence on field strength [23-28]. In these cases, there is a relatively small disparity between the tip and the issuing-jet diameters compared to classical electrospray, suggesting that this could play a relevant role in the effect of the external field. Consistent with this idea, recent theoretical work suggests that this size difference could indeed affect the local electric field around the area where the microjet forms, thereby affecting the current behavior [29]. However, despite the interest in achieving electric control over the current, as this would additionally provide a means to change the resultant drop sizes, the underlying reasons governing the field strength dependence of the current still remain unclear.

In this Letter, we use a glass-based microfluidic device in a novel way to quantify the behavior of current as a function of operating parameters and find that, unlike in classical electrospray, there is a strong voltage dependence and a weak flow rate dependence of the current. To understand these results, we consider that the current is dominated by convection near the apex of the cone and driven by tangential electric stresses rather than by the flow rate of the liquid. By writing a shear stress balance that accounts for this additional effect, we capture the order of magnitude of the measured current and quantitatively describe its voltage and flow rate dependence. Our results open the way to induce electric control over the current and, as a result, over the size of the drops formed in the process.

We use a microfluidic device made of glass similar to that used for engineering double emulsions [30]. It consists of a capillary with a square cross section of inner side $1 \mathrm{~mm}$ and two cylindrical capillaries of similar outer diameter, coaxially aligned with the square one. We pump a liquid of conductivity $K=10^{-4} \mathrm{~S} / \mathrm{m}$ and viscosity $\eta_{i}=17 \mathrm{cP}$ through one of these two capillaries, which was pulled to have an inner diameter $d_{i}=25 \mu \mathrm{m}$. Through the voids left between the outer square cross section and the inner circular cross section of this capillary, we pump a dielectric liquid of viscosity $\eta_{o}=10 \mathrm{cP}$, at a constant flow rate of $30 \mathrm{ml} / \mathrm{h}$. The third liquid, which we call a liquid collector, is also a conductor and flows through the inside of the second cylindrical capillary. As a result of the imposed flows, the exit of the device is provided by the voids left between the outer square cross section and the circular 
cross section of this second cylindrical capillary, as shown in Fig. 1(a). To apply the external electric field, we establish an electric potential difference between the metallic needles in contact with the inner liquid and liquid collector, which act as electrodes due to their finite conductivity.

Using this liquid configuration, we fix the flow rate of the inner liquid $q_{i}$ and adjust the flow rate of the liquid collector to maintain a constant tip-to-liquid-collector distance $L=900 \mu \mathrm{m}$. We then progressively increase the applied voltage $V$ and monitor the meniscus behavior by using high speed imaging and optical microscopy. In the absence of applied voltage, we observe the expected dripping behavior [31], where drops grow at the tip and detach when the drag exerted by the outer liquid overcomes surface tension forces; this is similar to the dripping at any faucet, except that it is driven by drag rather than by weight. For low voltages, the inner liquid continues to drip, but the resultant drop size decreases with increasing voltage as a result of the additional electric forces exerted on the drop [32,33]; this is often called the electrodripping regime. At even higher voltages, we observe a pulsating behavior, with the meniscus alternating between hemispherical and conical shapes [33,34]. This oscillatory behavior of the meniscus often precedes the steady-state generation of drops in electrospray. Consistent with this, by applying a slightly larger voltage, we observe the formation of a meniscus with a stable conical shape with a steady-state microjet issuing from its apex, as shown in Fig. 1(b). The microjet either directly breaks into drops due to the growth of axisymmetric hydrodynamic instabilities, as also shown in Fig. 1(b), or undergoes a 3-dimensional, nonaxisymmetric hydrodynamic instability at higher flow rates of the inner liquid before breaking into drops, as

(a)

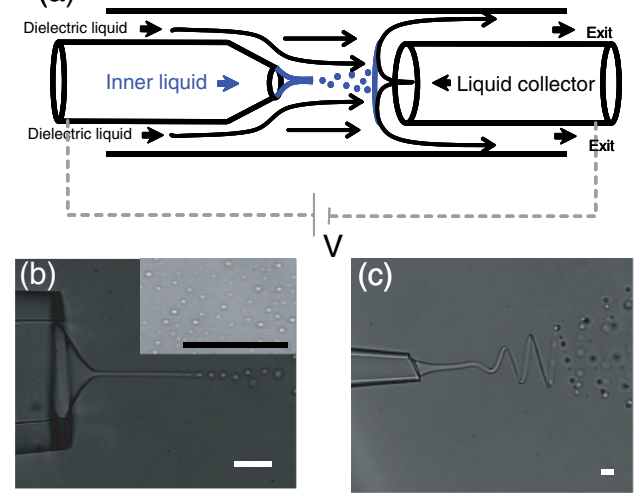

FIG. 1 (color online). (a) Schematic of the experimental device. The inner liquid is ethylene glycol, and the coflowing, dielectric liquid is a poly(dimethylsiloxane) oil. The interfacial tension is $\gamma=40 \mathrm{mN} / \mathrm{m}$. (b) Image corresponding to the conejet mode, with a jet issuing from the conical meniscus and breaking into drops. The inset shows an image of an extracted emulsion with average drop diameter $1 \mu \mathrm{m}$. (c) Image corresponding to the whipping mode, where the jet exhibits an offaxis, bending instability before it breaks into drops. The scale bar in all images is $20 \mu \mathrm{m}$. shown in Fig. 1(c). The first mode of drop formation essentially results from the Rayleigh-Plateau instability responsible for the breakup of water jets emanating from a faucet [35,36], although in this case there are fluid flow and surface charge involved [37]. The second mode is called whipping and results from the off-axis instability associated with charged jets $[5,25,38]$. If the growth rate of this mode is larger than the growth rate of the axisymmetric mode, the jet bends off-axis before it can break into drops.

The experimentally measured current is independent of the hydrodynamic instability associated with the microjet. This indicates that the mechanism of charge transport in our device is independent of the fate of the jet. We find that the current grows linearly with applied voltage, as shown in Fig. 2. This is significantly different from what is usually observed in most common electrospray processes, where the current is essentially independent of the applied voltage [17-22]. In these experiments, $d_{i}$ is several orders of magnitude larger than the jet diameter $d_{\text {jet }}$, implying that the electric field near the region where the microjet issues, which is typically a distance of $O\left(d_{i}\right)$ away from the capillary, very much depends on the charge distribution near the interface and not so much on the externally applied field. As a result, $I \neq I(V)$. In addition, near the issuing jet, the speed of the inner liquid is markedly larger than it is at the tip, implying that in this region the current is controlled by the convection of the surface charge, which is driven by the inner liquid flow rate. As a result, the current solely depends on $q_{i}$ [17].

In our experiments, $d_{i}$ is at most about an order of magnitude larger than $d_{\text {jet }}$, as is also the case in most situations where a voltage dependence of the current has been observed. As a result, the local field around the region where the microjet forms could be affected by the external field, which would still be significant at a distance of $O\left(d_{i}\right)$ away from the capillary. Based on the linear $I-V$ characteristic of

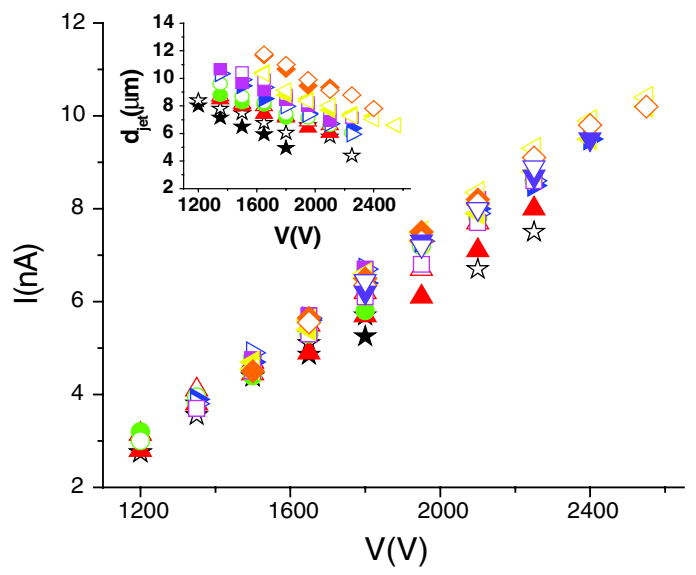

FIG. 2 (color online). Experimentally measured current and jet diameter (inset) as a function of applied voltage at different $q_{i}$ :

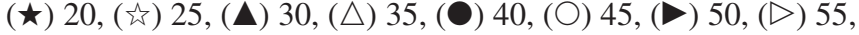

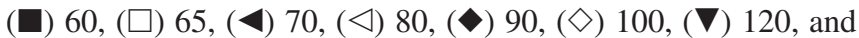
( $\nabla) 150 \mu \mathrm{l} / \mathrm{h}$. 
our device, this effect could reflect contributions to the current from conduction mechanisms. To test this possibility, we estimate the conduction current in the straight part of the microjet right after it forms: $I_{\text {cond }}=$ $2 \pi K \int_{0}^{d_{\mathrm{jet}} / 2} E_{x}^{i} r d r \approx \pi K \bar{E}_{x}^{i} d_{\text {jet }}^{2} / 4$, where $K$ is the electric conductivity of the inner liquid and $\bar{E}_{x}^{i}$ is the average axial component of the electric field inside the inner liquid, which we estimate considering that our electrode configuration resembles a needle-plate configuration, where $E=C V$ / $\left[z \ln \left(2 L / d_{i}\right)\right]$ [29], with $z$ the axial distance measured from the tip and $C$ a constant equal to 0.59 for our device geometry, as determined by computer simulations [39]. We find that the measured current scaled by this theoretical estimate increases with voltage, as shown in Fig. 3. The slope associated with this increase is larger than the slope associated with the $I-V$ characteristic, indicating that measured and estimated currents show opposite trends with applied voltage; while the measured current increases with voltage, as shown in Fig. 2, the estimated conduction current decreases with voltage. This decrease results from the reduction in $d_{\text {jet }}$ with voltage, as shown in the inset in Fig. 2. These results clearly rule out conduction as the responsible mechanism for the current increase we observe with voltage.

We thus hypothesize that the behavior is due to convection but driven by the external electric field rather than driven by the liquid flow rate. To test this, we evaluate the convective contribution to the current in the straight part of the microjet right after it forms: $I_{\text {conv }}=\pi d_{\text {jet }} \sigma u_{s}$, where $\sigma$ is the surface charge density, which results from the migration of charges to the interface in an attempt to decrease to zero the electric field inside the inner liquid and $u_{s}$ is the interface speed. We obtain this speed by writing a simple tangential stress balance at the jet surface $[40,41]$ :

$$
\frac{\mu_{i}\left(u_{i}-u_{s}\right)}{d_{\text {jet }}}+\sigma E_{t}=\frac{\mu_{o}\left(u_{s}-u_{o}\right)}{\delta},
$$

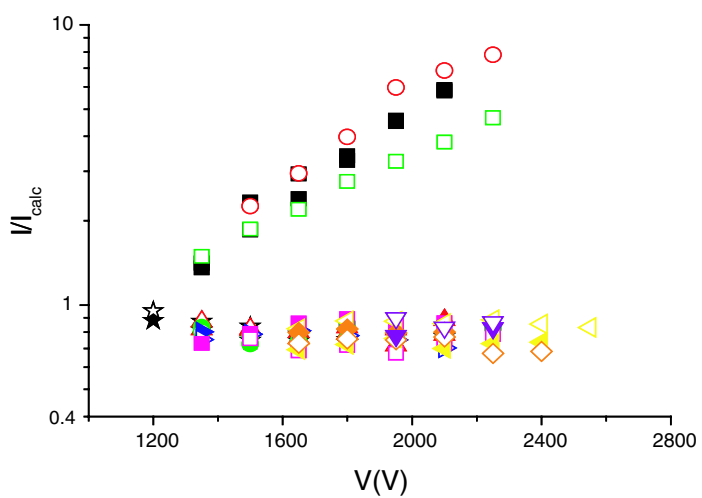

FIG. 3 (color online). Experimentally measured current scaled with calculated current, assuming conduction, as a function of applied voltage at different $q_{i}:(\square) 45$, (口) 60, and (○) $70 \mu \mathrm{l} / \mathrm{h}$. Experimentally measured current scaled with calculated current, assuming convection, as a function of applied voltage at different

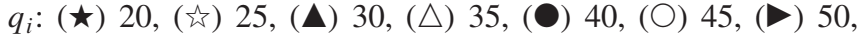

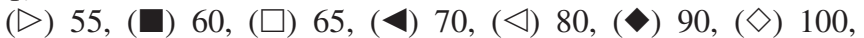
( $\nabla) 120$, and $(\nabla) 150 \mu \mathrm{l} / \mathrm{h}$. where $\mu_{o}$ and $\mu_{i}$ are the viscosities of the outer and inner liquids, respectively, $u_{o}$ and $u_{i}$ are the average velocities of the outer and inner liquids, respectively, $E_{t}$ is the tangential component of the electric field, and $\delta$ is the thickness of the shear layer outside the jet. The first and third terms in the stress balance are the viscous stresses exerted by the inner and outer liquids, respectively, on the surface of the jet, while the second term is the tangential electric stress on the jet surface. To estimate $\delta$, we approximate the jet by a cylinder and take into account the enhanced shear stress on a surface with this shape compared to the more familiar flat shape, which results in a reduced shear boundary layer of thickness $\delta \simeq d_{\text {jet }}$ [42]. For the surface charge density, we assume that the normal component of the electric field outside the jet is given by Taylor's field [43], since this field strength is what is at least required to induce the formation of a conical meniscus [13]. As a result, $\sigma=$ $\epsilon E_{n} \simeq \epsilon \sqrt{2 \gamma /\left(\epsilon d_{\text {jet }}\right)}$, with $\epsilon$ the permittivity of the outer dielectric liquid, $E_{n}$ the normal component of the electric field outside the jet, and $\gamma$ the interfacial tension. Finally, we estimate $E_{t}$ by considering the needle-plate expression evaluated in the straight part of the microjet right after it forms.

With all these ingredients, we solve for $u_{s}$ in Eq. (1) and consider the measured voltage and flow rate dependence of $d_{\text {jet }}$ to estimate the convection current. By doing so, we are able to capture the experimentally measured current dependence with voltage; the measured current normalized by this theoretical estimate does not depend on voltage and is of order 1, as shown in Fig. 3. We thus are able to describe the order of magnitude of the experimental measurements as well as their dependence with voltage, confirming our hypothesis.

Our model further predicts a weak $q_{i}$ dependence of the current, as shown in Fig. 4(a). This is in contrast to conventional electrospray, where $I \sim q_{i}^{1 / 2}[13,17]$. To see whether this prediction is consistent with our data, we measure the current as a function of $q_{i}$ at various voltages and normalize it with the theoretical expectation, as shown in Fig. 4(b). We see that this ratio is independent of $q_{i}$ and of order 1, emphasizing the agreement between our model and the experimental results. For other liquids and geometrical configurations, the results are similar and well described by our model [44].

The key to our data is the importance of the tangential electric stress. When $d_{i} / d_{\text {jet }}$ is not very large, we find that the electric field in the straight part of the jet right after it forms, at a distance $O\left(d_{i}\right)$ away from the capillary tip, given to a first approximation by that of the needle-plate configuration, is still large and able to affect the transport of surface charge. In classical electrospray, however, since $d_{i} / d_{\text {jet }}$ is very large, the imposed needle-plate field has significantly decreased near the region where the microjet forms and does not contribute to the surface charge transport; in this case, the charge is convected solely by the flow 

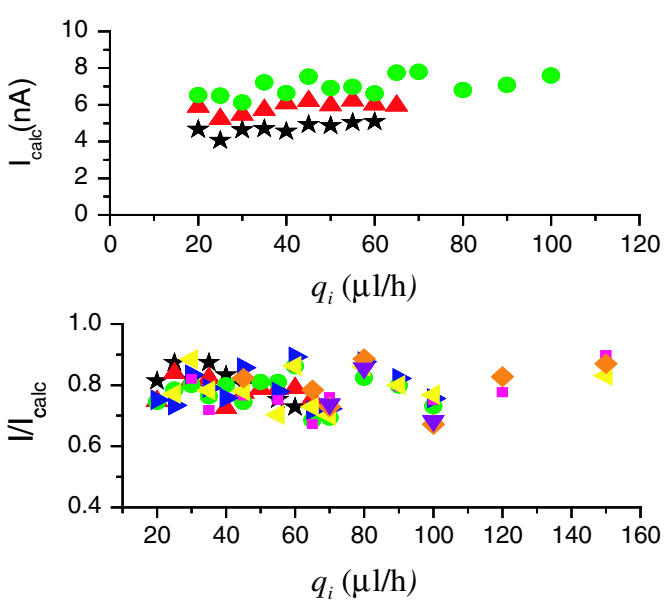

FIG. 4 (color online). (a) Calculated convection current as a function of $q_{i}$ for different applied voltages: ( $\star$ ) 1350, ( $\boldsymbol{\Delta}$ ) 1500, and (-) $1650 \mathrm{~V}$. (b) Experimentally measured current normalized with the calculated convection current versus $q_{i}$ for different

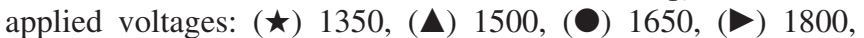

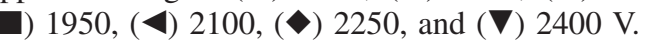

rate. As a result, the current in these situations is not affected by the voltage, and it depends only on the innerfluid flow rate. Decreasing this length-scale disparity changes the relative influence of the external field which at some point is able to affect the behavior of the electric current. This is consistent with other experiments [23-28], which could also be rationalized in terms of a stress balance similar to that of Eq. (1) [45]. In these cases, however, the viscous stress due to the outer flow is absent.

The glass-based microfluidic device used in the way shown here also allows the steady generation and extraction of drops with an average size that can be much smaller than the smallest geometrical feature of the device [inset in Fig. 1(b)]. We emphasize the key role played by the liquid collector; it neutralizes the electric charge on the drops and drags them out of the device, ensuring its steady-state operation. From a technical point of view, the use of liquid wires enables the application of electric fields without patterned electrodes or metallic components inside the device. This further allows the use of glass capillaries, which widens the selection of liquids that could be used compared to the more common polydimethylsiloxane devices. Our method thus opens new avenues to the use of electric fields in microfluidics for drop generation.

We thank DPI2008-06624-C03-03 and NSF (DMR0847304 and CBET-0967293). We are also thankful to Professors I. G. Loscertales, A. Barrero, and J. F. Higuera.

*Present address: Grupo de Quimica Coloidal e Interfacial, CSIC (Spain).

[1] J. Zeleny, Phys. Rev. 10, 1 (1917).

[2] A. Barrero and I. G. Loscertales, Annu. Rev. Fluid Mech. 39, 89 (2007).

[3] J. B. Fenn et al., Science 246, 64 (1989).
[4] W. Siefert, Thin Solid Films 120, 267 (1984).

[5] J. Doshi and D. H. Reneker, J. Electrost. 35, 151 (1995).

[6] Y. M. Shin et al., Polymer 42, 9955 (2001).

[7] D. H. Reneker et al., J. Appl. Phys. 87, 4531 (2000).

[8] D. Li and Y. Xia, Adv. Mater. 16, 1151 (2004).

[9] I. G. Loscertales et al., Science 295, 1695 (2002).

[10] E. Mathiowitz et al., Nature (London) 386, 410 (1997).

[11] A. Barrero et al., J. Colloid Interface Sci. 272, 104 (2004).

[12] H. Kim et al., Appl. Phys. Lett. 91, 133106 (2007).

[13] J. Fernandez de la Mora, Annu. Rev. Fluid Mech. 39, 217 (2007).

[14] G. I. Taylor, Proc. R. Soc. A 280, 383 (1964).

[15] I. Hayati et al., J. Colloid Interface Sci. 117, 222 (1987).

[16] R. T. Collins et al., Nature Phys. 4, 149 (2008).

[17] J. Fernandez de la Mora and I. G. Loscertales, J. Fluid Mech. 260, 155 (1994).

[18] A. M. Gañan-Calvo, Phys. Rev. Lett. 79, 217 (1997).

[19] A. M. Gañan-Calvo et al., J. Aerosol Sci. 28, 249 (1997).

[20] D. Chen and D. Y. H. Pui, Aerosol Sci. Technol. 27, 367 (1997).

[21] F. J. Higuera, J. Fluid Mech. 484, 303 (2003).

[22] A. M. Gañan-Calvo, J. Fluid Mech. 335, 165 (1997).

[23] M. S. Wilm and M. Mann, Int. J. Mass Spectrom. Ion Process. 136, 167 (1994).

[24] A. M. Gañan-Calvo et al., J. Fluid Mech. 566, 421 (2006).

[25] G. Riboux et al., J. Fluid Mech. (to be published).

[26] R. P. A. Hartman et al., J. Aerosol Sci. 30, 823 (1999).

[27] M. M. Hohman et al., Phys. Fluids 13, 2221 (2001).

[28] P. K. Bhattacharjee et al., J. Appl. Phys. 107, 044306 (2010).

[29] F. J. Higuera, J. Fluid Mech. 513, 239 (2004).

[30] A. S. Utada et al., Science 308, 537 (2005).

[31] A. S. Utada et al., Phys. Rev. Lett. 99, 094502 (2007).

[32] X. Zhang and O. A. Basaran, Phys. Fluids 7, 1184 (1995).

[33] M. Cloupeau and B. Prunet-Foch, J. Aerosol Sci. 25, 1021 (1994).

[34] R. Juraschek and F. W. Rollgen, Int. J. Mass Spectrom. 177, 1 (1998).

[35] J. Plateau, Acad. Sci. Bruxelles Mém. 23, 5 (1849).

[36] W. S. Rayleigh, Proc. London Math. Soc. s1-10, 4 (1878).

[37] R. T. Collins et al., J. Fluid Mech. 588, 75 (2007).

[38] S. V. Fridrikh et al., Phys. Rev. Lett. 90, 144502 (2003).

[39] We calculated $C$ for our experimental geometry by using the software COMSOL; we considered the presence of outer walls, the curvature of the counterelectrode, and the size of the tip, in the absence of fluid flow.

[40] J. R. Melcher and G. I. Taylor, Annu. Rev. Fluid Mech. 1, 111 (1969).

[41] D. A. Saville, Annu. Rev. Fluid Mech. 29, 27 (1997).

[42] M. B. Glauert and M. J. Lighthill, Proc. R. Soc. A 230, 188 (1955).

[43] The ratio of conduction time to residence time at the tip is $O(1)$, supporting the use of Taylor's field for estimating $E_{n}$.

[44] See supplementary material at http://link.aps.org/ supplemental/10.1103/PhysRevLett.105.154503 for other operating conditions.

[45] In recent experiments [28], it has been shown that the current has two contributions: It can be carried (i) by the jet and (ii) by secondary electrospray processes emanating from the jet itself. The field strength dependence, though, arises from the first contribution and is prone to the appropriate stress balance based on Eq. (1). 